Article

\title{
Stability of the Fréchet Equation in Quasi-Banach Spaces
}

\section{Sang Og Kim}

School of Data Science, Hallym University, Chuncheon 24252, Korea; sokim@hallym.ac.kr

Received: 24 February 2020; Accepted: 24 March 2020; Published: 1 April 2020

Abstract: We investigate the Hyers-Ulam stability of the well-known Fréchet functional equation that comes from a characterization of inner product spaces. We also show its hyperstability on a restricted domain. We work in the framework of quasi-Banach spaces. In the proof, a fixed point theorem due to Dung and Hang, which is an extension of a fixed point theorem in Banach spaces, plays a main role.

Keywords: Hyers-Ulam stability; hyperstability; Fréchet equation; quasi-Banach space; fixed point theorem

MSC: 39B52; 39B82; 47H10

\section{Introduction}

About eighty years ago, Ulam [1] raised a problem of finding conditions under which there exists an exact additive map near an approximate additive map. An answer to the problem between Banach spaces was given by Hyers [2]. After that, many authors have studied the stability problems. We refer to [3-7] for more information.

One of the most important outcomes of the stability of functional equations is the following theorem.

Theorem 1. Let $X, Y$ be two Banach spaces and $f: X \rightarrow Y$ be a mapping. Consider the following inequality

$$
\|f(x+y)-f(x)-f(y)\| \leq c\left(\|x\|^{p}+\|y\|^{p}\right),
$$

where $c>0$ and $p \neq 1$ are real constants. Then the following statements hold.

(i) If $p \geq 0$ and (1) holds for all $x, y \in X$, then there exists a unique additive mapping $T: X \rightarrow Y$ such that

$$
\|f(x)-T(x)\| \leq \frac{c}{\left|1-2^{p-1}\right|}\|x\|^{p} \text { for all } x \in X
$$

(ii) If $p<0$ and (1) holds for all $x, y \in X \backslash\{0\}$, then $f$ is additive.

The case $p=0$ is reduced to the stability by Hyers [2]. The case $0<p<1$ is due to Aoki [8] (see also [9]). Gajda [10] showed the stability of the Cauchy functional equation for $p>1$. Statement (ii) was proved first by Lee [11] and Brzdȩk [12] showed it on a restricted domain.

Let $G$ be an additive abelian group and let $Y$ be a linear space. We say that $f: G \rightarrow Y$ satisfies the Fréchet equation if

$$
f(x+y)+f(y+z)+f(x+z)=f(x+y+z)+f(x)+f(y)+f(z), \quad x, y, z \in G .
$$


The above equation was introduced by the classical equality

$$
\|x+y\|^{2}+\|y+z\|^{2}+\|x+z\|^{2}=\|x+y+z\|^{2}+\|x\|^{2}+\|y\|^{2}+\|z\|^{2}, \quad x, y, z \in Y
$$

in real or complex inner product spaces $Y$. In 1935, Fréchet [13] proved that in a normed space $Y,(3)$ is equivalent to the fact that $Y$ is an inner product space.

Recall that a map $q: G \rightarrow Y$ is said to be quadratic if it satisfies

$$
q(x+y)+q(x-y)=2 q(x)+2 q(y), \quad x, y \in G .
$$

It is known that every solution of (2) is of the form $f=a+q$, where $a: G \rightarrow Y$ is an additive mapping and $q: G \rightarrow Y$ is a quadratic mapping. (see, e.g., [14]). The stability of (2) in Banach spaces has been investigated by many authors (see, e.g., [15-22]). In particular, Bahyrycz et al. [15], Brzdęk et al. [16] and Malejki [21] have studied the generalized Fréchet functional equations with constant coefficients using a fixed point theorem in metric spaces by Brzdęk et al. [23].

In recent studies of the stability of functional equations, fixed point theorems play important roles. Dung and Hang [24] generalized the fixed point theorem of Brzdęk et al. [23] in metric spaces to $b$-metric spaces, and hence to quasi-Banach spaces. By using that fixed point theorem, they obtained a hyperstability of general linear equations. For more information on the stability of functional equations and fixed point theorems, we refer to $[25,26]$.

Several authors have studied the stability of many functional equations in quasi-Banach spaces (see, e.g., [24,27-32]).

The purpose of this paper is to obtain the (hyper)stability of (2) by using the fixed point theorem of Dung and Hang [24].

This paper is organized as follows.

In Section 2, we consider the hyperstability of (2) on a restricted domain. More precisly, let $X$ be a nonempty subset of a quasi-normed linear space and $Y$ be a quasi-Banach space. We say that a function $f: X \rightarrow Y$ satisfies the Fréchet equation on $X$ if

$$
f(x+y)+f(y+z)+f(x+z)=f(x+y+z)+f(x)+f(y)+f(z)
$$

for all $x, y, z \in X$ such that $x+y+z, x+y, y+z, x+z \in X$. We will show that Fréchet equation on $X$ is hyperstable; that is, if $f: X \rightarrow Y$ satisfies

$$
\|f(x+y)+f(y+z)+f(x+z)-f(x+y+z)-f(x)-f(y)-f(z)\| \leq c\left(\|x\|^{p}+\|y\|^{p}+\|z\|^{p}\right)
$$

for all $x, y, z$ in some set $X, p<0$ and $c \geq 0$, then $f$ must satisfy the Fréchet equation on $X$.

In Section 3, we consider the Hyers-Ulam stability results of (2) in quasi-Banach spaces. Especially, we investigate (4) for various $p \geq 0$.

In Section 4, we show that the Fréchet equation is not stable for $p=1,2$.

Throughout this paper, $\mathbb{N}$ stands for the set of all positive integers, $\mathbb{R}_{+}:=[0, \infty)$ and $A^{B}$ denotes the family of all functions mapping a set $B \neq \varnothing$ into a set $A \neq \varnothing$.

We recall some relevant notions of quasi-Banach spaces:

Definition 1. Let $X$ be a nonempty set, $\kappa \geq 1$ and $d: X \times X \rightarrow \mathbb{R}_{+}$be a function such that for all $x, y, z \in X$,

1. $d(x, y)=0$ if and only if $x=y$.

2. $d(x, y)=d(y, x)$.

3. $\quad d(x, z) \leq \kappa(d(x, y)+d(y, z))$.

Then

1. $d$ is called a b-metric on $X$ and $(X, d, \kappa)$ is called a $b$-metric space. 
2. The sequence $\left\{x_{n}\right\}$ is convergent to $x$ in $(X, d, \kappa)$ if $\lim _{n \rightarrow \infty} d\left(x_{n}, x\right)=0$.

3. The sequence $\left\{x_{n}\right\}$ is called a Cauchy sequence if $\lim _{n, m \rightarrow \infty} d\left(x_{n}, x_{m}\right)=0$.

4. The space $(X, d, \kappa)$ is said to be complete if each Cauchy sequence is convergent.

Definition 2. Let $X$ be a vector space over the field $\mathbb{K}=\mathbb{R}$ or $\mathbb{C}, \kappa \geq 1$ and $\|\cdot\|: X \times X \rightarrow \mathbb{R}_{+}$be a function such that for all $x, y, z \in X$ and all $a \in \mathbb{K}$,

1. $\|x\|=0$ if and only if $x=0$.

2. $|a x\|=|a|\| x \|$.

3. $\|x+y\| \leq \kappa(\|x\|+\|y\|)$.

Then $\|\cdot\|$ is called a quasi-norm on $X$ and $(X,\|\cdot\|, \kappa)$ is called a quasi-normed space.

Note that if $(X,\|\cdot\|, \kappa)$ is a quasi-normed space, letting $d(x, y)=\|x-y\|$ for $x, y \in X,(X, d, \kappa)$ becomes a $b$-metric space. Complete quasi-normed spaces are called quasi-Banach spaces.

A quasi-norm $\|\cdot\|$ is called a $p$-norm $(0<p \leq 1)$ if

$$
\|x+y\|^{p} \leq\|x\|^{p}+\|y\|^{p}, \quad x, y \in X
$$

In this case, we call the quasi-Banach space a $p$-Banach space. It is well-known that each quasi-norm is equivalent to some $p$-norm (see [33]). Since working with $p$-norms is much easier than working with quasi-norms, authors often restrict their attention to $p$-norms when they study the stability of functional equations between quasi-Banach spaces. However we will investigate the stability in quasi-Banach spaces with quasi-norms.

One of the most important class of quasi-Banach spaces is the class of $L^{p}(\mu)$ for $0<p<1$ with the usual quasi-norm

$$
\|f\|_{p}=\left(\int|f|^{p} d \mu\right)^{\frac{1}{p}}
$$

In this case,

$$
\|f+g\|_{p} \leq 2^{\frac{1}{p}-1}\left(\|f\|_{p}+\|g\|_{p}\right), \quad f, g \in L^{p}(\mu) .
$$

Hence, taking a particular case of $L^{p}(\mu)$, we have the following example.

Example 1. For $\left(x_{1}, x_{2}\right) \in \mathbb{R}^{2}$, define the quasi-norm of $\left(x_{1}, x_{2}\right)$ by

$$
\left\|\left(x_{1}, x_{2}\right)\right\|=\left(\sqrt{\left|x_{1}\right|}+\sqrt{\left|x_{2}\right|}\right)^{2} .
$$

Then $\left(\mathbb{R}^{2},\|\cdot\|, 2\right)$ is a quasi-Banach space.

The following lemma can be seen easily from 3 of Definition 2.

Lemma 1 ([31]). Let $(X,\|\cdot\|, \kappa)$ be a quasi-normed space and $x_{1}, \ldots, x_{2 n+1} \in X$. Then

$$
\left\|\sum_{j=1}^{2 n} x_{j}\right\| \leq \kappa^{n} \sum_{j=1}^{2 n}\left\|x_{j}\right\|, \quad\left\|\sum_{j=1}^{2 n+1} x_{j}\right\| \leq \kappa^{n+1} \sum_{j=1}^{2 n+1}\left\|x_{j}\right\| .
$$

\section{Hyperstability of (2) on a Restricted Domain}

The following theorem, which is a generalization of the outcome of [23], is the main tool in proving the results of this paper.

Theorem 2 ([24], Corollary 2.2). Suppose that 
1. $X$ is a nonempty set, $(Y,\|\cdot\|, \kappa)$ is a quasi-Banach space, and $J: Y^{X} \rightarrow Y^{X}$ is a given function.

2. There exist $f_{1}, \ldots, f_{n}: X \rightarrow X$ and $L_{1}, \ldots, L_{n}: X \rightarrow \mathbb{R}_{+}$such that for every $\xi, \mu \in Y^{X}, x \in X$,

$$
\|J \xi(x)-J \mu(x)\| \leq \sum_{i=1}^{n} L_{i}(x)\left\|\xi\left(f_{i}(x)\right)-\mu\left(f_{i}(x)\right)\right\| .
$$

3. There exist $\epsilon: X \rightarrow \mathbb{R}_{+}$and $\phi: X \rightarrow Y$ such that for all $x \in X$,

$$
\|J \phi(x)-\phi(x)\| \leq \epsilon(x) .
$$

4. For every $x \in X$ and $\theta=\log _{2 \kappa} 2$,

$$
\epsilon^{*}(x):=\sum_{n=0}^{\infty}\left(\Lambda^{n} \epsilon\right)^{\theta}(x)<\infty
$$

where

$$
\Lambda \delta(x)=\sum_{i=1}^{n} L_{i}(x) \delta\left(f_{i}(x)\right)
$$

for all $\delta \in \mathbb{R}_{+}^{X}$ and $x \in X$.

Then we have

1. For every $x \in X$, the limit

$$
\lim _{n \rightarrow \infty} J^{n} \phi(x)=\psi(x),
$$

exists and the function $\psi: X \rightarrow Y$ so defined is a fixed point of J satisfying

$$
\|\phi(x)-\psi(x)\|^{\theta} \leq 4 \epsilon^{*}(x)
$$

for all $x \in X$.

2. For every $x \in X$, if

$$
\epsilon^{*}(x) \leq\left(M \sum_{n=0}^{\infty}\left(\Lambda^{n} \epsilon\right)(x)\right)^{\theta}<\infty
$$

for some positive real number $M$, then the fixed point of J satisfying (10) is unique.

Now we state the main result of this section. Note that the domain of the mapping $f$ is a subset of a quasi-normed space that is not necessarily a commutative group. We adapt some ideas from [34,35]. Throughout this section, we denote $X:=X_{0} \backslash\{0\}$ for a subset $(0 \in) X_{0}$ of a quasi-Banach space.

Theorem 3. Assume that $X_{0}$ is a nonempty subset of a quasi-normed space such that $0 \in X_{0}=-X_{0}$ and there exists $n_{0} \in \mathbb{N}$ with $n x \in X_{0}$ for all $x \in X_{0}$ and for all $n \geq n_{0}$. Let $(Y,\|\cdot\|, \kappa)$ be a quasi-Banach space, $p<0$ and $c \geq 0$. If $f: X_{0} \rightarrow Y$ is a mapping that satisfies $f(0)=0$ and

$$
\|f(x+y+z)+f(x)+f(y)+f(z)-f(x+y)-f(y+z)-f(x+z)\| \leq c\left(\|x\|^{p}+\|y\|^{p}+\|z\|^{p}\right)
$$

for all $x, y, z \in X$ such that $x+y+z, x+y, y+z, x+z \in X_{0}$, then $f$ satisfies the Fréchet equation on $X$.

Proof. First observe that $\lim _{m \rightarrow \infty} m^{p}=0$, so there exists an integer $m_{0}$ such that

$$
\kappa^{2}\left(2(m+1)^{p}+2 m^{p}+(2 m+1)^{p}\right)<1 \text { for } m \geq m_{0} .
$$


Let us fix $m \geq \max \left\{n_{0}, m_{0}\right\}$. Replacing $(x, y, z)$ with $((m+1) x, m x,-m x)$ in (12), we have

$$
\|2 f((m+1) x)+f(m x)+f(-m x)-f((2 m+1) x)-f(x)\| \leq c\left((m+1)^{p}+2 m^{p}\right)\|x\|^{p}
$$

for all $x \in X$.

Consider the mappings $J: Y^{X} \rightarrow Y^{X}$ and $\epsilon: X \rightarrow \mathbb{R}_{+}$given by

$$
J \xi(x)=2 \xi((m+1) x)+\xi(m x)+\xi(-m x)-\xi((2 m+1) x), \quad \xi \in Y^{X}, x \in X,
$$

and

$$
\epsilon(x)=c\left((m+1)^{p}+2 m^{p}\right)\|x\|^{p}, \quad x \in X .
$$

The inequality (13) then becomes

$$
\|J f(x)-f(x)\| \leq \epsilon(x), \quad x \in X,
$$

so that (6) holds true. For every $\xi, \mu \in Y^{X}$ and $x \in X$, we have by Lemma 1

$$
\begin{aligned}
& \|J \xi(x)-J \mu(x)\| \\
& \leq \kappa^{2}(2\|(\xi-\mu)((m+1) x)\|+\|(\xi-\mu)(m x)\|+\|(\xi-\mu)(-m x)\|+\|(\xi-\mu)((2 m+1) x)\|) \\
& =\sum_{i=1}^{4} L_{i}(x)\left\|(\xi-\mu)\left(f_{i} x\right)\right\|,
\end{aligned}
$$

so that $J$ satisfies (5) with $f_{1}(x)=(m+1) x, f_{2}(x)=m x, f_{3}(x)=-m x, f_{4}(x)=(2 m+1) x, L_{1}(x)=2 \kappa^{2}$, and $L_{2}(x)=L_{3}(x)=L_{4}(x)=\kappa^{2}$.

Let $\Lambda: \mathbb{R}_{+}^{X} \rightarrow \mathbb{R}_{+}^{X}$ be given by

$$
\Lambda \eta(x)=2 \kappa^{2} \eta((m+1) x)+\kappa^{2} \eta(m x)+\kappa^{2} \eta(-m x)+\kappa^{2} \eta((2 m+1) x), \quad \eta \in \mathbb{R}_{+}^{X}, x \in X .
$$

Then

$$
\begin{aligned}
\Lambda \epsilon(x) & =\kappa^{2}(2 \epsilon((m+1) x)+\epsilon(m x)+\epsilon(-m x)+\epsilon((2 m+1) x)) \\
& =\kappa^{2}\left(2(m+1)^{p}+2 m^{p}+(2 m+1)^{p}\right) \epsilon(x), \quad x \in X .
\end{aligned}
$$

Since $\Lambda$ is linear, we have by induction

$$
\Lambda^{n} \epsilon(x)=\left[\kappa^{2}\left(2(m+1)^{p}+2 m^{p}+(2 m+1)^{p}\right)\right]^{n}\left[c\left((m+1)^{p}+2 m^{p}\right)\|x\|^{p}\right], \quad n \in \mathbb{N}, x \in X .
$$

Hence, noting that $0<\theta=\log _{2 \kappa} 2 \leq 1$, it follows that

$$
\begin{aligned}
\epsilon^{*}(x) & =\sum_{n=0}^{\infty}\left(\Lambda^{n} \epsilon\right)^{\theta}(x) \\
& =\sum_{n=0}^{\infty}\left[\kappa^{2}\left(2(m+1)^{p}+2 m^{p}+(2 m+1)^{p}\right)\right]^{n \theta}\left[c\left((m+1)^{p}+2 m^{p}\right)\|x\|^{p}\right]^{\theta} \\
& =\frac{\left[c\left((m+1)^{p}+2 m^{p}\right)\|x\|^{p}\right]^{\theta}}{1-\left[\kappa^{2}\left(2(m+1)^{p}+2 m^{p}+(2 m+1)^{p}\right)\right]^{\theta}}, \quad x \in X .
\end{aligned}
$$

Thus, by Theorem 2, there is a solution $F: X \rightarrow Y$ of the equation

$$
2 F((m+1) x)+F(m x)+F(-m x)-F((2 m+1) x)=F(x)
$$


such that

$$
\|f(x)-F(x)\|^{\theta} \leq \frac{4\left[c\left((m+1)^{p}+2 m^{p}\right)\|x\|^{p}\right]^{\theta}}{1-\left[\kappa^{2}\left(2(m+1)^{p}+2 m^{p}+(2 m+1)^{p}\right)\right]^{\theta}}, \quad x \in X .
$$

Moreover,

$$
F(x)=\lim _{n \rightarrow \infty} J^{n} f(x), \quad x \in X .
$$

To prove that $F$ satisfies the Fréchet equation on $X$, observe that

$$
\begin{aligned}
\left\|J^{n} f(x+y+z)+J^{n} f(x)+J^{n} f(y)+J^{n} f(z)-J^{n} f(x+y)-J^{n} f(y+z)-J^{n} f(x+z)\right\| \\
\leq c\left[\kappa^{2}\left(2(m+1)^{p}+2 m^{p}+(2 m+1)^{p}\right)\right]^{n}\left(\|x\|^{p}+\|y\|^{p}+\|z\|^{p}\right)
\end{aligned}
$$

for all $x, y, z \in X$ such that $x+y+z, x+y, y+z, x+z \in X$. In fact, this can be obtained from (12) by induction on $n \in \mathbb{N}$.

Letting $n \rightarrow \infty$ in (20), it follows from (19) that

$$
F(x+y+z)+F(x)+F(y)+F(z)-F(x+y)-F(y+z)-F(x+z)=0
$$

for all $x, y, z \in X$ such that $x+y+z, x+y, y+z, x+z \in X$.

Until now, we have proved that for every integer $m \geq \max \left\{n_{0}, m_{0}\right\}$, there exists a mapping $F_{m}: X \rightarrow Y$ satisfying

$$
F_{m}(x+y+z)+F_{m}(x)+F_{m}(y)+F_{m}(z)-F_{m}(x+y)-F_{m}(y+z)-F_{m}(x+z)=0,
$$

for all $x, y, z \in X$ such that $x+y+z, x+y, y+z, x+z \in X$, and

$$
\left\|f(x)-F_{m}(x)\right\|^{\theta} \leq \frac{4\left[c\left((m+1)^{p}+2 m^{p}\right)\|x\|^{p}\right]^{\theta}}{1-\left[\kappa^{2}\left(2(m+1)^{p}+2 m^{p}+(2 m+1)^{p}\right)\right]^{\theta}}
$$

for all $x \in X$.

Now, we show that $F_{m}=F_{k}$ for all $m, k \geq \max \left\{m_{0}, n_{0}\right\}$. Fix $m, k \geq \max \left\{m_{0}, n_{0}\right\}$ and denote $\epsilon_{m}(x)=c\left((m+1)^{p}+2 m^{p}\right)\|x\|^{p}$ and $\epsilon_{k}(x)=c\left((k+1)^{p}+2 k^{p}\right)\|x\|^{p}$ for all $x \in X$.

By (21), we get

$$
\begin{aligned}
& \left\|F_{m}(x)-F_{k}(x)\right\| \\
& \leq \frac{\kappa 4^{\frac{1}{\theta}} \epsilon_{m}(x)}{\left[1-\left[\kappa^{2}\left(2(m+1)^{p}+2 m^{p}+(2 m+1)^{p}\right)\right]^{\theta}\right]^{\frac{1}{\theta}}} \\
& \quad+\frac{\kappa 4^{\frac{1}{\theta}} \epsilon_{k}(x)}{\left[1-\left[\kappa^{2}\left(2(k+1)^{p}+2 k^{p}+(2 k+1)^{p}\right)\right]^{\theta}\right]^{\frac{1}{\theta}}} .
\end{aligned}
$$


Noting that $F_{m}$ and $F_{k}$ are fixed points of $J$, and $\Lambda$ is linear, we have by (16) and (22)

$$
\begin{aligned}
& \left\|F_{m}(x)-F_{k}(x)\right\| \\
& =\left\|J^{n} F_{m}(x)-J^{n} F_{k}(x)\right\| \\
& \leq \frac{\kappa 4^{\frac{1}{\theta}} \Lambda^{n} \epsilon_{m}(x)}{\left[1-\left[\kappa^{2}\left(2(m+1)^{p}+2 m^{p}+(2 m+1)^{p}\right)\right]^{\theta}\right]^{\frac{1}{\theta}}} \\
& \quad+\frac{\kappa 4^{\frac{1}{\theta}} \Lambda^{n} \epsilon_{k}(x)}{\left[1-\left[\kappa^{2}\left(2(k+1)^{p}+2 k^{p}+(2 k+1)^{p}\right)\right]^{\theta}\right]^{\frac{1}{\theta}}} \\
& =\frac{\kappa 4^{\frac{1}{\theta}}\left[\kappa^{2}\left(2(m+1)^{p}+2 m^{p}+(2 m+1)^{p}\right)\right]^{n} \epsilon_{m}(x)}{\left[1-\left[\kappa^{2}\left(2(m+1)^{p}+2 m^{p}+(2 m+1)^{p}\right)\right]^{\theta}\right]^{\frac{1}{\theta}}} \\
& \quad+\frac{\kappa 4^{\frac{1}{\theta}}\left[\kappa^{2}\left(2(k+1)^{p}+2 k^{p}+(2 k+1)^{p}\right)\right]^{n} \epsilon_{k}(x)}{\left[1-\left[\kappa^{2}\left(2(k+1)^{p}+2 k^{p}+(2 k+1)^{p}\right)\right]^{\theta}\right]^{\frac{1}{\theta}}} \\
& \rightarrow 0 \text { as } n \rightarrow \infty .
\end{aligned}
$$

Hence $F_{m}=F_{k}$ and we denote it by $F:=F_{m}=F_{k}$. Then, by (21), it follows that

$$
\|f(x)-F(x)\| \leq \frac{4^{\frac{1}{\theta}} c\left((m+1)^{p}+2 m^{p}\right)\|x\|^{p}}{\left[1-\left[\kappa^{2}\left(2(m+1)^{p}+2 m^{p}+(2 m+1)^{p}\right)\right]^{\theta}\right]^{\frac{1}{\theta}}}
$$

for all $x \in X$. Since $p<0$, the right hand side of (23) tends to zero as $m \rightarrow \infty$. Hence, we conclude that $f(x)=F(x)$ for all $x \in X$. Therefore, $f$ satisfies the Fréchet equation on $X$, completing the proof.

Notice that the assumption of unboundedness of $X$ is indispensable.

Example 2. Let $X_{0}=[-1,1], \mathbb{R}^{2}$ be the quasi-Banach space in Example 1 and $f: X_{0} \rightarrow \mathbb{R}^{2}$ be defined by $f(x)=(|x|, 0), x \in X_{0}$. Then for all $x, y, z \in X$ such that $x+y+z, x+y, y+z, x+z \in X$,

$$
\|f(x+y+z)+f(x)+f(y)+f(z)-f(x+y)-f(y+z)-f(x+z)\| \leq 3\left(|x|^{p}+|y|^{p}+|z|^{p}\right)
$$

for $p<0$. However $f$ does not satisfy the Fréchet equation on $X$.

In the case of $p \geq 0$, the Fréchet equation is not hyperstable.

Remark 1. Let $X=\mathbb{R} \backslash[-1,1], Y$ be a quasi-Banach space and let $f: X \rightarrow Y$ be a constant function $f(x)=c, x \in X$ for some $c \neq 0 \in Y$ and $p \geq 0$. Then $f$ satisfies

$$
\|f(x+y+z)+f(x)+f(y)+f(z)-f(x+y)-f(y+z)-f(x+z)\| \leq\|c\|\left(|x|^{p}+|y|^{p}+|z|^{p}\right)
$$

for all $x, y, z \in X$ such that $x+y+z, x+y, y+z, x+z \in X$. However $f$ does not satisfy the Fréchet equation on $X$.

\section{Stability of (2) on Abelian Groups}

In this section, we investigate the stability of (2) and as byproducts we get stability results of (4) for various $p \geq 0$ similar to Theorem 1 (see Corollaries 2, 3 and 4 below). 
Lemma 2. Let $G$ be an additive abelian group and $Y$ be a linear space. If $f: G \rightarrow Y$ is a mapping satisfying (2) with $f(2 x)=2 f(x)$ for all $x \in G$, then $f$ is additive.

Proof. We first note that $f(0)=0$. Replacing $(x, y, z)$ with $(x, x,-x)$ in (2), we have

$$
3 f(x)+f(-x)=f(2 x)=2 f(x), \quad x \in G,
$$

and hence,

$$
f(-x)=-f(x), \quad x \in G .
$$

Replacing $(x, y, z)$ with $(x, y,-y)$ in (2), we get

$$
f(x+y)+f(x-y)=2 f(x), \quad x, y \in G .
$$

Replacing $(x, y)$ with $(y, x)$ in $(24)$, we have

$$
f(x+y)+f(y-x)=2 f(y), \quad x, y \in G .
$$

Adding (24) and (25), we obtain

$$
f(x+y)=f(x)+f(y), \quad x, y \in G .
$$

Lemma 3. Let $G$ be an additive abelian group and $Y$ be a linear space. If $f: G \rightarrow Y$ is a mapping satisfying (2) with $f(2 x)=4 f(x)$ for all $x \in G$, then $f$ is quadratic.

Proof. Replacing $(x, y, z)$ with $(x, x,-x)$ in (2), we have

$$
3 f(x)+f(-x)=f(2 x)=4 f(x), \quad x \in G,
$$

and hence,

$$
f(-x)=f(x), \quad x \in G .
$$

Replacing $(x, y, z)$ with $(x, y,-y)$ in (2), we get

$$
f(x+y)+f(x-y)=2 f(x)+f(y)+f(-y)=2 f(x)+2 f(y), \quad x, y \in G .
$$

Hence, by definition, $f$ is quadratic.

Theorem 4. Assume that $(X,+)$ is an abelian group, $(Y,\|\cdot\|, \kappa)$ is a quasi-Banach space and $L<1$ is a real number such that $0<\frac{\kappa}{3}(2 L+1)<1$. Let $\varphi: X^{3} \rightarrow \mathbb{R}_{+}$be a function such that

$$
\varphi(2 x, 2 y, 2 z) \leq 2 L \varphi(x, y, z), \quad \varphi(x, y, z)=\varphi(-x,-y,-z), \quad x, y, z \in X
$$

If $f: X \rightarrow Y$ is a mapping that satisfies $f(0)=0$ and

$$
\|f(x+y+z)+f(x)+f(y)+f(z)-f(x+y)-f(y+z)-f(x+z)\| \leq \varphi(x, y, z)
$$

for all $x, y, z \in X$, then there exists a unique mapping $g: X \rightarrow Y$ satisfying (2) such that

$$
\|f(x)-g(x)\| \leq \frac{4^{\frac{1}{\theta}}}{\left[3^{\theta}-\kappa^{\theta}(2 L+1)^{\theta}\right]^{\frac{1}{\theta}}} \varphi(x, x,-x), \quad x \in X .
$$


Proof. Replacing $(x, y, z)$ with $(x, x,-x)$ in (26), we have

$$
\|f(2 x)-3 f(x)-f(-x)\| \leq \varphi(x, x,-x), \quad x \in X,
$$

so that

$$
\left\|\frac{1}{3} f(2 x)-\frac{1}{3} f(-x)-f(x)\right\| \leq \frac{1}{3} \varphi(x, x,-x), \quad x \in X .
$$

Consider the mappings $J: Y^{X} \rightarrow Y^{X}$ and $\epsilon: X \rightarrow \mathbb{R}_{+}$given by

$$
J \xi(x)=\frac{1}{3} \xi(2 x)-\frac{1}{3} \xi(-x), \quad \xi \in Y^{X}, x \in X,
$$

and

$$
\epsilon(x)=\frac{1}{3} \varphi(x, x,-x), \quad x \in X
$$

The inequality (28) becomes

$$
\|J f(x)-f(x)\| \leq \epsilon(x), \quad x \in X,
$$

so that (6) holds true. For every $\xi, \eta \in Y^{X}$ and $x \in X$, we have

$$
\|J \xi(x)-J \eta(x)\| \leq \frac{\kappa}{3}\|\xi(2 x)-\eta(2 x)\|+\frac{\kappa}{3}\|\xi(-x)-\eta(-x)\|,
$$

and hence, $J$ satisfies (5) with $f_{1}(x)=2 x, f_{2}(x)=-x$ and $L_{1}(x)=L_{2}(x)=\frac{\kappa}{3}$.

Let $\Lambda: \mathbb{R}_{+}^{X} \rightarrow \mathbb{R}_{+}^{X}$ be given by

$$
\Lambda \eta(x)=\kappa\left(\frac{1}{3} \eta(2 x)+\frac{1}{3} \eta(-x)\right), \quad \eta \in \mathbb{R}_{+}^{X}, x \in X .
$$

Then we have

$$
\Lambda \epsilon(x)=\kappa\left(\frac{1}{3} \epsilon(2 x)+\frac{1}{3} \epsilon(-x)\right) \leq \frac{\kappa}{3}(2 L+1) \epsilon(x), \quad x \in X .
$$

Note that $\Lambda$ is order-preserving, that is, if $\xi(x) \geq \eta(x)$ for all $x \in X$, then

$$
\Lambda \xi(x)=\Lambda \xi(x)-\Lambda \eta(x)+\Lambda \eta(x)=\Lambda(\xi-\eta)(x)+\Lambda \eta(x) \geq \Lambda \eta(x) .
$$

Hence, we have for all $n \in \mathbb{N}$

$$
\Lambda^{n} \epsilon(x) \leq\left(\kappa \frac{2 L+1}{3}\right)^{n} \epsilon(x), \quad x \in X
$$

As $\frac{\kappa(2 L+1)}{3}<1$ and $0<\theta=\log _{2 \kappa} 2 \leq 1$, we obtain

$$
\begin{aligned}
\epsilon^{*}(x) & =\sum_{n=0}^{\infty}\left(\Lambda^{n} \epsilon\right)^{\theta}(x) \leq \sum_{n=0}^{\infty}\left(\frac{\kappa(2 L+1)}{3}\right)^{n \theta} \epsilon^{\theta}(x) \\
& =\frac{1}{1-\left(\frac{\kappa(2 L+1)}{3}\right)^{\theta}}\left(\frac{1}{3} \varphi(x, x,-x)\right)^{\theta}=\frac{1}{3^{\theta}-\kappa^{\theta}(2 L+1)^{\theta}} \varphi(x, x,-x)^{\theta}, \quad x \in X .
\end{aligned}
$$

Therefore, by Theorem 2 , there exists a mapping $g: X \rightarrow Y$ such that

$$
\begin{aligned}
& g(x)=\lim _{n \rightarrow \infty} J^{n} f(x), \quad x \in X, \\
& g(x)=\frac{1}{3} g(2 x)-\frac{1}{3} g(-x), \quad x \in X,
\end{aligned}
$$


and

$$
\|f(x)-g(x)\|^{\theta} \leq \frac{4}{3^{\theta}-\kappa^{\theta}(2 L+1)^{\theta}} \varphi(x, x,-x)^{\theta}, \quad x \in X,
$$

from which inequality (27) follows.

Now we show that $g$ satisfies (2). From (26) and the definition of $J$, we have

$$
\begin{aligned}
& \|J f(x+y+z)+J f(x)+J f(y)+J f(z)-J f(x+y)-J f(y+z)-J f(x+z)\| \\
& \leq \frac{\kappa}{3} \varphi(2 x, 2 y, 2 z)+\frac{\kappa}{3} \varphi(-x,-y,-z) \\
& \leq \frac{\kappa(2 L+1)}{3} \varphi(x, y, z), \quad x \in X .
\end{aligned}
$$

By induction, we have for all $n \in \mathbb{N}$,

$$
\begin{aligned}
\left\|J^{n} f(x+y+z)+J^{n} f(x)+J^{n} f(y)+J^{n} f(z)-J^{n} f(x+y)-J^{n} f(y+z)-J^{n} f(x+z)\right\| & \\
& \leq\left(\frac{\kappa(2 L+1)}{3}\right)^{n} \varphi(x, y, z), \quad x, y, z \in X .
\end{aligned}
$$

Therefore, letting $n \rightarrow \infty$ in (30), we get

$$
g(x+y+z)+g(x)+g(y)+g(z)-g(x+y)-g(y+z)-g(x+z)=0, \quad x, y, z \in X .
$$

Next, we show the uniqueness of $g$. Assume that $g_{1}, g_{2}: X \rightarrow Y$ are mappings satisfying (2) and

$$
\left\|f(x)-g_{i}(x)\right\|^{\theta} \leq \frac{4}{3^{\theta}-\kappa^{\theta}(2 L+1)^{\theta}} \varphi(x, x,-x)^{\theta}, \quad i=1,2, x \in X .
$$

Then, by inequality 3 in Definition 2,

$$
\left\|g_{1}(x)-g_{2}(x)\right\| \leq \frac{2 \cdot 4^{\frac{1}{\theta}} \mathcal{K}}{\left[3^{\theta}-\kappa^{\theta}(2 L+1)^{\theta}\right]^{\frac{1}{\theta}}} \varphi(x, x,-x), \quad x \in X .
$$

Note by (29) that

$$
g_{i}(x)=\frac{1}{3} g_{i}(2 x)-\frac{1}{3} g_{i}(-x), \quad i=1,2, x \in X
$$

Then

$$
\begin{aligned}
& \left\|g_{1}(x)-g_{2}(x)\right\| \\
& =\left\|\frac{1}{3}\left(g_{1}(2 x)-g_{2}(2 x)\right)-\frac{1}{3}\left(g_{1}(-x)-g_{2}(-x)\right)\right\| \\
& \leq \frac{\kappa}{3} \frac{2 \cdot 4^{\frac{1}{\theta}} \mathcal{L}}{\left[3^{\theta}-\kappa^{\theta}(2 L+1)^{\theta}\right]^{\frac{1}{\theta}}} \varphi(2 x, 2 x,-2 x) \\
& \quad+\frac{\kappa}{3} \frac{2 \cdot 4^{\frac{1}{\theta}} \mathcal{K}}{\left[3^{\theta}-\kappa^{\theta}(2 L+1)^{\theta}\right]^{\frac{1}{\theta}}} \varphi(-x,-x, x) \\
& \leq \frac{\kappa(2 L+1)}{3} \frac{2 \cdot 4^{\frac{1}{\theta}} \mathcal{L}}{\left[3^{\theta}-\kappa^{\theta}(2 L+1)^{\theta}\right]^{\frac{1}{\theta}}} \varphi(x, x,-x), \quad x \in X .
\end{aligned}
$$


Applying the same argument repeatedly, it is easy to show that for all $n \in \mathbb{N}$,

$$
\left\|g_{1}(x)-g_{2}(x)\right\| \leq\left[\frac{\kappa(2 L+1)}{3}\right]^{n} \frac{2 \cdot 4^{\frac{1}{\theta}} \mathcal{K}}{\left[3^{\theta}-\kappa^{\theta}(2 L+1)^{\theta}\right]^{\frac{1}{\theta}}} \varphi(x, x,-x), \quad x \in X .
$$

Letting $n \rightarrow \infty$ in (31), we obtain $g_{1}=g_{2}$, as desired.

Putting $\varphi(x, y, z) \equiv c$, we have the following classical Ulam stability of the functional equation under consideration.

Corollary 1. Assume that $(X,+)$ is an abelian group, $(Y,\|\cdot\|, \kappa)$ is a quasi-Banach space with $\kappa<\frac{3}{2}$ and $c \geq 0$ is a constant. If $f: X \rightarrow Y$ is a mapping that satisfies $f(0)=0$ and

$$
\|f(x+y+z)+f(x)+f(y)+f(z)-f(x+y)-f(y+z)-f(x+z)\| \leq c
$$

for all $x, y, z \in X$, then there exists a unique mapping $g: X \rightarrow Y$ satisfying (2) such that

$$
\|f(x)-g(x)\| \leq \frac{4^{\frac{1}{\theta}} \mathcal{C}}{\left[3^{\theta}-2^{\theta} \kappa^{\theta}\right]^{\frac{1}{\theta}}}, \quad x \in X .
$$

Proof. We use Theorem 4 applied with $L=\frac{1}{2}$ and $\varphi(x, y, z)=c$ for all $x, y, z \in X$.

As an example of Theorem 4, we have the following stability of (4) for $0<p<1$.

Corollary 2. Let $(X,+)$ be an abelian subgroup of a quasi-normed space and $(Y,\|\cdot\|, \kappa)$ be a quasi-Banach space. Assume that, for some $0<p<1$ and some $c>0$, the mapping $f: X \rightarrow Y$ satisfies

$$
\|f(x+y+z)+f(x)+f(y)+f(z)-f(x+y)-f(y+z)-f(x+z)\| \leq c\left(\|x\|^{p}+\|y\|^{p}+\|z\|^{p}\right),
$$

for all $x, y, z \in X$. If $1 \leq \kappa<\frac{3}{2^{p-1}+1}$, then there exists a unique mapping $g: X \rightarrow Y$ satisfying (2) such that

$$
\|f(x)-g(x)\| \leq \frac{3 \cdot 4^{\frac{1}{\theta}} \mathcal{L}}{\left[3^{\theta}-\kappa^{\theta}\left(2^{p}+1\right)^{\theta}\right]^{\frac{1}{\theta}}}\|x\|^{p}, \quad x \in X .
$$

Proof. Taking $L=2^{p-1}$ in Theorem 4 , we obtain the result.

Recall that an abelian group $(X,+)$ is called uniquely 2-divisible if for each $x \in X$, there exists a unique $y \in X$ such that $2 y=x$. We denote $y=\frac{x}{2}$.

Theorem 5. Assume that $(X,+)$ is a uniquely 2-divisible abelian group, $(Y,\|\cdot\|, \kappa)$ is a quasi-Banach space and $0<L<\frac{1}{\kappa}$ is a real number. Let $\varphi: X^{3} \rightarrow \mathbb{R}_{+}$be a function such that

$$
\varphi(x, y, z) \leq \frac{L}{4} \varphi(2 x, 2 y, 2 z), \quad \varphi(x, y, z)=\varphi(-x,-y,-z)
$$

for all $x, y, z \in X$. If $f: X \rightarrow Y$ is a mapping that satisfies

$$
\|f(x+y+z)+f(x)+f(y)+f(z)-f(x+y)-f(y+z)-f(x+z)\| \leq \varphi(x, y, z)
$$

for all $x, y, z \in X$, then there exists a unique mapping $g: X \rightarrow Y$ satisfying (2) such that

$$
\|f(x)-g(x)\| \leq \frac{L}{4} \frac{4^{\frac{1}{\theta}}}{\left[1-(\kappa L)^{\theta}\right]^{\frac{1}{\theta}}} \varphi(x, x,-x), \quad x \in X .
$$


Proof. We first note that $f(0)=0$. Replacing $(x, y, z)$ with $\left(\frac{x}{2}, \frac{x}{2},-\frac{x}{2}\right)$ in (32), we have

$$
\left\|f(x)-3 f\left(\frac{x}{2}\right)-f\left(-\frac{x}{2}\right)\right\| \leq \varphi\left(\frac{x}{2}, \frac{x}{2},-\frac{x}{2}\right), \quad x \in X .
$$

Consider the mappings $J: Y^{X} \rightarrow Y^{X}$ and $\epsilon: X \rightarrow \mathbb{R}_{+}$given by

$$
J \xi(x)=3 \xi\left(\frac{x}{2}\right)+\xi\left(-\frac{x}{2}\right), \quad \xi \in Y^{X}, x \in X,
$$

and

$$
\epsilon(x)=\varphi\left(\frac{x}{2}, \frac{x}{2},-\frac{x}{2}\right), \quad x \in X
$$

Then inequality (34) becomes

$$
\|J f(x)-f(x)\| \leq \epsilon(x), \quad x \in X,
$$

so that (6) holds true. For every $\xi, \eta \in Y^{X}$ and $x \in X$, we have

$$
\|J \xi(x)-J \eta(x)\| \leq 3 \kappa\left\|\xi\left(\frac{x}{2}\right)-\eta\left(\frac{x}{2}\right)\right\|+\kappa\left\|\xi\left(-\frac{x}{2}\right)-\eta\left(-\frac{x}{2}\right)\right\|,
$$

so that $J$ satisfies (5) with $f_{1}(x)=\frac{x}{2}, f_{2}(x)=-\frac{x}{2}, L_{1}(x)=3 \kappa$ and $L_{2}(x)=\kappa$.

Let $\Lambda: \mathbb{R}_{+}^{X} \rightarrow \mathbb{R}_{+}^{X}$ be given by

$$
\Lambda \eta(x)=3 \kappa \eta\left(\frac{x}{2}\right)+\kappa \eta\left(-\frac{x}{2}\right), \quad \eta \in \mathbb{R}_{+}^{X}, x \in X .
$$

Then we have

$$
\begin{aligned}
\Lambda \epsilon(x) & =3 \kappa \epsilon\left(\frac{x}{2}\right)+\kappa \epsilon\left(-\frac{x}{2}\right)=3 \kappa \varphi\left(\frac{x}{2^{2}}, \frac{x}{2^{2}},-\frac{x}{2^{2}}\right)+\kappa \varphi\left(-\frac{x}{2^{2}},-\frac{x}{2^{2}}, \frac{x}{2^{2}}\right) \\
& =4 \kappa \varphi\left(\frac{x}{2^{2}}, \frac{x}{2^{2}},-\frac{x}{2^{2}}\right)=4 \kappa \epsilon\left(\frac{x}{2}\right), \quad x \in X .
\end{aligned}
$$

By induction on $n$, we get

$$
\Lambda^{n} \epsilon(x)=4^{n} \kappa^{n} \epsilon\left(\frac{x}{2^{n}}\right), \quad x \in X,
$$

and hence

$$
\begin{aligned}
\epsilon^{*}(x) & =\sum_{n=0}^{\infty}\left(\Lambda^{n} \epsilon\right)^{\theta}(x)=\sum_{n=0}^{\infty}\left(4^{n} \kappa^{n}\right)^{\theta} \varphi\left(\frac{x}{2^{n+1}}, \frac{x}{2^{n+1}},-\frac{x}{2^{n+1}}\right)^{\theta} \\
& \leq \sum_{n=0}^{\infty} 4^{n \theta} \kappa^{n \theta}\left(\frac{L}{4}\right)^{(n+1) \theta} \varphi(x, x,-x)^{\theta}=\left(\frac{L}{4}\right)^{\theta} \frac{1}{1-(\kappa L)^{\theta}} \varphi(x, x,-x)^{\theta}, \quad x \in X,
\end{aligned}
$$

so that (7) holds true. Therefore, by Theorem 2, there exists a mapping $g: X \rightarrow Y$ such that

$$
\begin{aligned}
& g(x)=\lim _{n \rightarrow \infty} J^{n} f(x), \quad x \in X, \\
& g(x)=3 g\left(\frac{x}{2}\right)+g\left(-\frac{x}{2}\right), \quad x \in X,
\end{aligned}
$$

and

$$
\|f(x)-g(x)\|^{\theta} \leq\left(\frac{L}{4}\right)^{\theta} \frac{4}{1-(\kappa L)^{\theta}} \varphi(x, x,-x)^{\theta}, \quad x \in X .
$$

Inequality (33) follows from (36) 
Now we show that $g$ satisfies (2). From (32) and the definition of $J$, we have

$$
\begin{aligned}
& \|J f(x+y+z)+J f(x)+J f(y)+J f(z)-J f(x+y)-J f(y+z)-J f(x+z)\| \\
& \leq 3 \kappa \varphi\left(\frac{x}{2}, \frac{y}{2}, \frac{z}{2}\right)+\kappa \varphi\left(-\frac{x}{2},-\frac{y}{2},-\frac{z}{2}\right) \\
& =4 \kappa \varphi\left(\frac{x}{2}, \frac{y}{2}, \frac{z}{2}\right) \\
& \leq \kappa L \varphi(x, y, z), \quad x \in X .
\end{aligned}
$$

By induction, we have for all $n \in \mathbb{N}$ and $x, y, z \in X$,

$$
\begin{aligned}
& \left\|J^{n} f(x+y+z)+J^{n} f(x)+J^{n} f(y)+J^{n} f(z)-J^{n} f(x+y)-J^{n} f(y+z)-J^{n} f(x+z)\right\| \\
& \leq(\kappa L)^{n} \varphi(x, y, z) .
\end{aligned}
$$

Therefore, letting $n \rightarrow \infty$ in (37), we obtain

$$
g(x+y+z)+g(x)+g(y)+g(z)-g(x+y)-g(y+z)-g(x+z)=0, \quad x, y, z \in X .
$$

Next, we show the uniqueness of $g$. Assume that $g_{1}, g_{2}: X \rightarrow Y$ are mappings satisfying (2) and

$$
\left\|f(x)-g_{i}(x)\right\| \leq \frac{L}{4} \frac{4^{\frac{1}{\theta}}}{\left[1-(\kappa L)^{\theta}\right]^{\frac{1}{\theta}}} \varphi(x, x,-x), \quad i=1,2, x \in X .
$$

Then

$$
\left\|g_{1}(x)-g_{2}(x)\right\| \leq \frac{\kappa L}{2} \frac{4^{\frac{1}{\theta}}}{\left[1-(\kappa L)^{\theta}\right]^{\frac{1}{\theta}}} \varphi(x, x,-x), \quad x \in X .
$$

By (35), we have

$$
g_{i}(x)=3 g_{i}\left(\frac{x}{2}\right)+g_{i}\left(-\frac{x}{2}\right), \quad i=1,2, x \in X .
$$

Hence

$$
\begin{aligned}
& \left\|g_{1}(x)-g_{2}(x)\right\| \\
& =3 \kappa\left\|g_{1}\left(\frac{x}{2}\right)-g_{2}\left(\frac{x}{2}\right)\right\|+\kappa\left\|g_{1}\left(-\frac{x}{2}\right)-g_{2}\left(-\frac{x}{2}\right)\right\| \\
& \leq \frac{\kappa L}{2} \frac{4^{\frac{1}{\theta}}}{\left[1-(\kappa L)^{\theta}\right]^{\frac{1}{\theta}}}\left(3 \kappa \varphi\left(\frac{x}{2}, \frac{x}{2},-\frac{x}{2}\right)+\kappa \varphi\left(-\frac{x}{2},-\frac{x}{2}, \frac{x}{2}\right)\right) \\
& \leq \frac{(\kappa L)^{2}}{2} \frac{4^{\frac{1}{\theta}}}{\left[1-(\kappa L)^{\theta}\right]^{\frac{1}{\theta}}} \varphi(x, x,-x), \quad x \in X .
\end{aligned}
$$

In this way, it is easy to show that for all $n \in \mathbb{N}$,

$$
\left\|g_{1}(x)-g_{2}(x)\right\| \leq \frac{(\kappa L)^{n}}{2} \frac{4^{\frac{1}{\theta}}}{\left[1-(\kappa L)^{\theta}\right]^{\frac{1}{\theta}}} \varphi(x, x,-x), \quad x \in X .
$$

Letting $n \rightarrow \infty$ in (38), it follows that $g_{1}=g_{2}$. This completes the proof.

As an application of Theorem 5, we have the following stability of (4) for $p>2$. 
Corollary 3. Let $(X,+)$ be a uniquely 2-divisible abelian subgroup of a quasi-normed space and $(Y,\|\cdot\|, \kappa)$ be a quasi-Banach space. Assume $f: X \rightarrow Y$ is a mapping that satisfies

$$
\|f(x+y+z)+f(x)+f(y)+f(z)-f(x+y)-f(y+z)-f(x+z)\| \leq c\left(\|x\|^{p}+\|y\|^{p}+\|z\|^{p}\right),
$$

for $p>2, c>0$ and for all $x, y, z \in X$. If $1 \leq \kappa<2^{p-2}$, then there exists a unique mapping $g: X \rightarrow Y$ satisfying (2) such that

$$
\|f(x)-g(x)\| \leq \frac{3 \cdot 2^{-p} \cdot 4^{\frac{1}{\theta}} \cdot c}{\left[1-\left(2^{2-p_{\mathcal{K}}}\right)^{\theta}\right]^{\frac{1}{\theta}}}\|x\|^{p}, \quad x \in X .
$$

Proof. Taking $L=2^{2-p}$ and applying Theorem 5, we get the result.

Theorem 6. Assume that $(X,+)$ is a uniquely 2-divisible abelian group, $(Y,\|\cdot\|, \kappa)$ is a quasi-Banach space and $L<1$ is a real number. Let $\varphi: X^{3} \rightarrow \mathbb{R}_{+}$be a function such that

$$
\varphi(2 x, 2 y, 2 z) \leq 4 L \varphi(x, y, z), \varphi(x, y, z) \leq \frac{L}{2} \varphi(2 x, 2 y, 2 z), \varphi(x, y, z)=\varphi(-x,-y,-z)
$$

for all $x, y, z \in X$. If $f: X \rightarrow Y$ is a mapping that satisfies

$$
\|f(x+y+z)+f(x)+f(y)+f(z)-f(x+y)-f(y+z)-f(x+z)\| \leq \varphi(x, y, z)
$$

for all $x, y, z \in X$, then there exist a unique additive mapping $g_{0}: X \rightarrow Y$ and a unique quadratic mapping $g_{e}: X \rightarrow Y$ such that

$$
\left\|f(x)-g_{o}(x)-g_{e}(x)\right\| \leq \frac{4^{\frac{1}{\theta}-1} \kappa^{2}(1+2 L)}{\left(1-L^{\theta}\right)^{\frac{1}{\theta}}} \varphi(x, x,-x), \quad x \in X .
$$

Proof. Note first that $f(0)=0$. Let $f_{e}: X \rightarrow Y$ and $f_{0}: X \rightarrow Y$ be the even and odd parts of $f$, respectively. That is, $f_{e}(x)=\frac{f(x)+f(-x)}{2}, f_{o}(x)=\frac{f(x)-f(-x)}{2}$ for $x \in X$. Then $f_{e}(0)=f_{o}(0)=0$. It is easy to show that

$$
\left\|f_{e}(x+y+z)+f_{e}(x)+f_{e}(y)+f_{e}(z)-f_{e}(x+y)-f_{e}(y+z)-f_{e}(x+z)\right\| \leq \kappa \varphi(x, y, z)
$$

and analogously,

$$
\left\|f_{o}(x+y+z)+f_{o}(x)+f_{o}(y)+f_{o}(z)-f_{o}(x+y)-f_{o}(y+z)-f_{o}(x+z)\right\| \leq \kappa \varphi(x, y, z),
$$

for all $x, y, z \in X$. Replacing $(x, y, z)$ with $(x, x,-x)$ in (41), we have

$$
\left\|f_{e}(2 x)-3 f_{e}(x)-f_{e}(-x)\right\| \leq \kappa \varphi(x, x,-x),
$$

so that

$$
\left\|f_{e}(2 x)-4 f_{e}(x)\right\| \leq \kappa \varphi(x, x,-x), \quad x \in X
$$

Hence, it follows that

$$
\left\|\frac{1}{4} f_{e}(2 x)-f_{e}(x)\right\| \leq \frac{1}{4} \kappa \varphi(x, x,-x), \quad x \in X .
$$


As before, define mappings $J, \Lambda$ and $\epsilon$ by

$$
\begin{aligned}
J \xi(x) & =\frac{1}{4} \xi(2 x), \quad \xi \in Y^{X}, \quad x \in X, \\
\Lambda \delta(x) & =\frac{1}{4} \delta(2 x), \quad \delta \in \mathbb{R}_{+}^{X}, \quad x \in X, \\
\epsilon(x) & =\frac{1}{4} \kappa \varphi(x, x,-x), \quad x \in X .
\end{aligned}
$$

Then, we have by (43)

$$
\left\|J f_{e}(x)-f_{e}(x)\right\| \leq \epsilon(x)
$$

so that (6) holds true.

For every $\xi, \eta \in Y^{X}$ and $x \in X$, we have

$$
\|J \xi(x)-J \eta(x)\| \leq \frac{1}{4}\|(\xi(2 x)-\eta(2 x))\|,
$$

from which $J$ satisfies (5) with $f_{1}(x)=2 x$ and $L_{1}(x)=\frac{1}{4}$. Note that

$$
\begin{aligned}
\Lambda^{n} \epsilon(x) & =\frac{1}{4^{n}} \epsilon\left(2^{n} x\right) \\
& =\frac{1}{4^{n+1}} \kappa \varphi\left(2^{n} x, 2^{n} x,-2^{n} x\right) \\
& \leq \frac{1}{4^{n+1}}(4 L)^{n} \kappa \varphi(x, x,-x)=\frac{\kappa}{4} L^{n} \varphi(x, x,-x), \quad x \in X .
\end{aligned}
$$

Hence, we get

$$
\epsilon^{*}(x)=\sum_{n=0}^{\infty}\left(\Lambda^{n} \epsilon\right)^{\theta}(x) \leq \frac{\left(\frac{\kappa}{4}\right)^{\theta}}{1-L^{\theta}} \varphi(x, x,-x)^{\theta}, \quad x \in X,
$$

so that (7) holds true. Therefore, by Theorem 2 there exists a mapping $g_{e}: X \rightarrow Y$ such that

$$
\begin{aligned}
& g_{e}(x)=\lim _{n \rightarrow \infty} J^{n} f_{e}(x), \quad x \in X, \\
& g_{e}(x)=\frac{1}{4} g_{e}(2 x), \quad x \in X,
\end{aligned}
$$

and

$$
\left\|g_{e}(x)-f_{e}(x)\right\|^{\theta} \leq \frac{4\left(\frac{\kappa}{4}\right)^{\theta}}{1-L^{\theta}} \varphi(x, x,-x)^{\theta}, \quad x \in X .
$$

Since, by (41)

$$
\begin{aligned}
& \| J^{n} f_{e}(x+y+z)+J^{n} f_{e}(x)+J^{n} f_{e}(y)+J^{n} f_{e}(z)-J^{n} f_{e}(x+y) \\
& \quad \quad-J^{n} f_{e}(y+z)-J^{n} f_{e}(x+z) \| \\
& =\frac{1}{4^{n}} \| f_{e}\left(2^{n}(x+y+z)\right)+f_{e}\left(2^{n} x\right)+f_{e}\left(2^{n} y\right)+f_{e}\left(e^{n} z\right) \\
& \quad \quad-f_{e}\left(2^{n}(x+y)\right)-f_{e}\left(2^{n}(y+z)\right)-f_{e}\left(2^{n}(x+z)\right) \| \\
& \leq \frac{\kappa}{4^{n}} \varphi\left(2^{n} x, 2^{n} y, 2^{n} z\right) \\
& \leq \frac{\kappa}{4^{n}}(4 L)^{n} \varphi(x, y, z)=\kappa L^{n} \varphi(x, y, z), \quad x \in X,
\end{aligned}
$$

it follows that $g_{e}$ satisfies (2). Then, on account of Lemma 3 and (44), we infer that $g_{e}$ is a quadratic mapping. 
We apply a similar argument to the mapping $f_{0}$. Replacing $(x, y, z)$ with $(x, x,-x)$ in (42), we have

$$
\left\|f_{o}(2 x)-2 f_{o}(x)\right\| \leq \kappa \varphi(x, x,-x), \quad x \in X
$$

Replacing $x$ with $\frac{x}{2}$ in (46), we have

$$
\left\|f_{o}(x)-2 f_{o}\left(\frac{x}{2}\right)\right\| \leq \kappa \varphi\left(\frac{x}{2}, \frac{x}{2},-\frac{x}{2}\right), \quad x \in X .
$$

Let

$$
\begin{gathered}
J \xi(x)=2 \xi\left(\frac{x}{2}\right), \quad \xi \in Y^{X}, \quad x \in X, \\
\Lambda \delta(x)=2 \delta\left(\frac{x}{2}\right), \quad \delta \in \mathbb{R}_{+}^{X}, \quad x \in X, \\
\epsilon(x)=\kappa \varphi\left(\frac{x}{2}, \frac{x}{2},-\frac{x}{2}\right), \quad x \in X .
\end{gathered}
$$

Then, it follows by (47)

$$
\left\|J f_{o}(x)-f_{o}(x)\right\| \leq \epsilon(x)
$$

so that (6) holds true.

For every $\xi, \eta \in Y^{X}$ and $x \in X$, we have

$$
\|J \xi(x)-J \eta(x)\| \leq 2\left\|\xi\left(\frac{x}{2}\right)-\eta\left(\frac{x}{2}\right)\right\|,
$$

from which $J$ satisfies (5) with $f_{1}(x)=\frac{x}{2}$ and $L_{1}(x)=2$. Note that

$$
\begin{aligned}
\Lambda^{n} \epsilon(x) & =2^{n} \epsilon\left(\frac{x}{2^{n}}\right) \\
& =2^{n} \kappa \varphi\left(\frac{x}{2^{n+1}}, \frac{x}{2^{n+1}},-\frac{x}{2^{n+1}}\right) \\
& \leq 2^{n} \kappa\left(\frac{L}{2}\right)^{n+1} \varphi(x, x,-x)=\frac{\kappa L}{2} \cdot L^{n} \varphi(x, x,-x), \quad x \in X .
\end{aligned}
$$

Hence

$$
\begin{aligned}
\epsilon^{*}(x) & =\sum_{n=0}^{\infty}\left(\Lambda^{n} \epsilon\right)^{\theta}(x) \leq \sum_{n=0}^{\infty}\left(\frac{\kappa L}{2}\right)^{\theta} \cdot L^{n \theta} \varphi(x, x,-x)^{\theta} \\
& =\left(\frac{\kappa L}{2}\right)^{\theta} \frac{1}{1-L^{\theta}} \varphi(x, x,-x)^{\theta}, \quad x \in X,
\end{aligned}
$$

so that (7) holds true. Therefore, by Theorem 2, there exists a mapping $g_{o}: X \rightarrow Y$ such that

$$
\begin{aligned}
& g_{o}(x)=\lim _{n \rightarrow \infty} J^{n} f_{o}(x), \quad x \in X, \\
& g_{o}(x)=2 g_{o}\left(\frac{x}{2}\right), \quad x \in X,
\end{aligned}
$$

and

$$
\left\|g_{o}(x)-f_{o}(x)\right\|^{\theta} \leq\left(\frac{\kappa L}{2}\right)^{\theta} \frac{4}{1-L^{\theta}} \varphi(x, x,-x)^{\theta}, \quad x \in X .
$$


Since, by (42)

$$
\begin{aligned}
& \| J^{n} f_{o}(x+y+z)+J^{n} f_{o}(x)+J^{n} f_{o}(y)+J^{n} f_{o}(z)-J^{n} f_{o}(x+y) \\
& -J^{n} f_{o}(y+z)-J^{n} f_{o}(x+z) \| \\
& =2^{n} \| f_{o}\left(\frac{x+y+z}{2^{n}}\right)+f_{o}\left(\frac{x}{2^{n}}\right)+f_{o}\left(\frac{y}{2^{n}}\right)+f_{o}\left(\frac{z}{2^{n}}\right) \\
& -f_{o}\left(\frac{x+y}{2^{n}}\right)-f_{o}\left(\frac{y+z}{2^{n}}\right)-f_{o}\left(\frac{x+z}{2^{n}}\right) \| \\
& \leq 2^{n} \kappa \varphi\left(\frac{x}{2^{n}}, \frac{y}{2^{n}}, \frac{z}{2^{n}}\right) \leq 2^{n} \kappa\left(\frac{L}{2}\right)^{n} \varphi(x, y, z)=\kappa L^{n} \varphi(x, y, z)
\end{aligned}
$$

for all $x, y, z \in X$, it follows that $g_{o}$ satisfies (2). Then by Lemma 2 and (48), we infer that $g_{o}$ is an additive mapping. Thus $g=g_{e}+g_{o}$ also satisfies (2).

By (45) and (49), we obtain

$$
\begin{aligned}
\|f(x)-g(x)\| & \leq \kappa\left(\left\|f_{e}(x)-g_{e}(x)\right\|+\left\|f_{o}(x)-g_{o}(x)\right\|\right) \\
& \leq \kappa\left[\frac{4^{\frac{1}{\theta}} \frac{\kappa}{4}}{\left(1-L^{\theta}\right)^{\frac{1}{\theta}}}+\left(\frac{\kappa L}{2}\right) \frac{4^{\frac{1}{\theta}}}{\left(1-L^{\theta}\right)^{\frac{1}{\theta}}}\right] \varphi(x, x,-x) \\
& =\frac{4^{\frac{1}{\theta}-1} \kappa^{2}(1+2 L)}{\left(1-L^{\theta}\right)^{\frac{1}{\theta}}} \varphi(x, x,-x), \quad x \in X,
\end{aligned}
$$

as desired. Finally, we show the uniqueness. Assume there exists another additive mapping $g_{0}^{\prime}: X \rightarrow Y$ and a quadratic mapping $g_{e}^{\prime}: X \rightarrow Y$ such that

$$
\left\|f(x)-g_{o}^{\prime}(x)-g_{e}^{\prime}(x)\right\| \leq \frac{4^{\frac{1}{\theta}-1} \kappa^{2}(1+2 L)}{\left(1-L^{\theta}\right)^{\frac{1}{\theta}}} \varphi(x, x,-x), \quad x \in X .
$$

Letting $\alpha:=\frac{4^{\frac{1}{\theta}-1} \kappa^{2}(1+2 L)}{\left(1-L^{\theta}\right)^{\frac{1}{\theta}}}$, and taking the even part of the mapping $f-g_{o}-g_{e}$ (resp. $\left.f-g_{o}^{\prime}-g_{e}^{\prime}\right)$, we have from (39)

$$
\left\|f_{e}(x)-g_{e}(x)\right\| \leq \kappa \alpha \varphi(x, x,-x) \text { and }\left\|f_{e}(x)-g_{e}^{\prime}(x)\right\| \leq \kappa \alpha \varphi(x, x,-x), \quad x \in X .
$$

Then

$$
\begin{aligned}
\left\|g_{e}(x)-g_{e}^{\prime}(x)\right\| & =\frac{1}{4}\left\|g_{e}(2 x)-g_{e}^{\prime}(2 x)\right\| \\
& \leq \frac{1}{4} \kappa^{2} \alpha \varphi(2 x, 2 x,-2 x) \leq \frac{1}{4} \kappa^{2} \alpha \cdot 4 L \varphi(x, x,-x) \\
& =L \kappa^{2} \alpha \varphi(x, x-x), \quad x \in X .
\end{aligned}
$$

In this manner, we get for all $n \in \mathbb{N}$

$$
\left\|g_{e}(x)-g_{e}^{\prime}(x)\right\| \leq L^{n} \kappa^{2} \alpha \varphi(x, x-x),
$$

which goes to zero as $n \rightarrow \infty$. Hence $g_{e}=g_{e}^{\prime}$. Similarly, we can show that $g_{o}=g_{o}^{\prime}$.

Applying Theorem 6, we have the following stability of (4) for $1<p<2$. 
Corollary 4. Assume that $(X,+)$ is a uniquely 2-divisible abelian subgroup of a quasi-normed space and $(Y,\|\cdot\|, \kappa)$ is a quasi-Banach space. Let the constants $1<p<2$ and $c>0$ be such that the mapping $f: X \rightarrow Y$ satisfies

$$
\|f(x+y+z)+f(x)+f(y)+f(z)-f(x+y)-f(y+z)-f(x+z)\| \leq c\left(\|x\|^{p}+\|y\|^{p}+\|z\|^{p}\right),
$$

for all $x, y, z \in X$. Then there exist a unique additive mapping $g_{o}: X \rightarrow Y$ and a unique quadratic mapping $g_{e}: X \rightarrow Y$ such that

$$
\left\|f(x)-g_{o}(x)-g_{e}(x)\right\| \leq \frac{3 c \cdot 4^{\frac{1}{\theta}-1} \kappa^{2}(1+2 L)}{\left(1-L^{\theta}\right)^{\frac{1}{\theta}}}\|x\|^{p}, \quad x \in X,
$$

where $L=\max \left\{2^{p-2}, 2^{1-p}\right\}$.

\section{Nonstability of the Fréchet Equation}

In this part, we show that the Fréchet equation is not stable for $p \in\{1,2\}$. The following example comes from Gajda [10].

Example 3. Let $\phi: \mathbb{R} \rightarrow \mathbb{R}$ be the function defined by

$$
\phi(x)= \begin{cases}-\alpha, & x \leq-1 \\ \alpha x, & -1<x<1 \\ \alpha, & 1 \leq x\end{cases}
$$

where $\alpha>0$. Then the function $f: \mathbb{R} \rightarrow \mathbb{R}$ given by

$$
f(x)=\sum_{n=0}^{\infty} \frac{\phi\left(2^{n} x\right)}{2^{n}}, \quad x \in \mathbb{R}
$$

satisfies

$$
|f(x+y+z)+f(x)+f(y)+f(z)-f(x+y)-f(y+z)-f(x+z)| \leq 14 \alpha(|x|+|y|+|z|),
$$

but there is no function g satisfying (2) with $c>0$ such that

$$
|f(x)-g(x)| \leq c|x|, \quad x \in \mathbb{R} .
$$

Proof. Following the proof of [10] with $|x|+|y|+|z|$ instead of $|x|+|y|$, we easily get the result.

For $p=2$, we consider the following example coming from [36].

Example 4. Let $\phi: \mathbb{R} \rightarrow \mathbb{R}$ be the function defined by

$$
\phi(x)= \begin{cases}\alpha, & x \in(-\infty,-1] \cup[1, \infty), \\ \alpha x^{2}, & -1<x<1,\end{cases}
$$

where $\alpha>0$. Then the function $f: \mathbb{R} \rightarrow \mathbb{R}$ given by

$$
f(x)=\sum_{n=0}^{\infty} \frac{\phi\left(2^{n} x\right)}{4^{n}}, \quad x \in \mathbb{R}
$$


satisfies

$$
|f(x+y+z)+f(x)+f(y)+f(z)-f(x+y)-f(y+z)-f(x+z)| \leq 40 \alpha\left(|x|^{2}+|y|^{2}+|z|^{2}\right)
$$

but there is no function $g$ satisfying (2) with c $>0$ such that

$$
|f(x)-g(x)| \leq c|x|^{2}, \quad x \in \mathbb{R}
$$

Proof. Following the proof of [36] with $x^{2}+y^{2}+z^{2}$ instead of $x^{2}+y^{2}$ and using the fact that $f$ is an even function, it is easy to get the result.

\section{Conclusions}

Using a recently developed fixed point theorem, we have proved the Hyers-Ulam stability of the Fréchet equation in quasi-Banach spaces. We also have shown the hyper-stability of the equation on a restricted domain. The method and results in this paper extend the existing ones in the literature mentioned in the Introduction.

Funding: This work was supported by Hallym University Research Fund, 2020 (HRF-202002-017).

Conflicts of Interest: The author declares no conflict of interest.

\section{References}

1. Ulam, S.M. Problems of Modern Mathematics; Sciences Editions; John Wiley \& Sons Inc.: New York, NY, USA, 1964.

2. Hyers, D.H. On the stability of linear functional equations. Proc. Natl. Acad. Sci. USA 1941, 27, $222-224$. [CrossRef] [PubMed]

3. Brzdęk, J.; Popa, D.; Raşa, I.; Xu, B. Ulam Stability of Operators; Academic Press: London, UK, 2018.

4. Czerwik, S. Functional Equations and Inequalities in Several Variables; World Scientific: River Edge, NY, USA, 2002.

5. Hyers, D.H.; Isac, G.; Rassias, T.M. Stability of Functional Equations in Several Variables; Birkhäuser: Basel, Germany, 1998.

6. Jung, S.M. Hyers-Ulam-Rassis Stability of Functional Equations in Mathematical Analysis; Springer: New York, NY, USA, 2011.

7. Rassias, T.M.; Brzdęk, J. (Eds.) Functional Equations in Mathematical Analysis; Springer: New York, NY, USA, 2012.

8. Aoki, T. On the stability of the linear transformation mappings in Banach spaces. J. Math. Soc. Jpn. 1950, 2, 64-66. [CrossRef]

9. Rassias, T.M. On the stability of linear mappings in Banach spaces. Proc. Am. Math. Soc. 1978, 72, $297-300$. [CrossRef]

10. Gajda, Z. On stability of additive mappings. Int. J. Math. Math. Sci. 1991, 14, 431-434. [CrossRef]

11. Lee, Y.-H. On the stability of the monomial functional equation. Bull. Korean Math. Soc. 2008, 45, $397-403$. [CrossRef]

12. Brzdęk, J. Hyperstability of the Cauchy equation on restricted domains. Acta Math. Hungar. 2013, $141,58-67$. [CrossRef]

13. Fréchet, M. Sur la définition axiomatique d'une classe d'espaces vectoriels distanciés applicables vectoriellement sur l'espace de Hilbert. Ann. Math. Second Ser. 1935, 36, 705-718. [CrossRef]

14. Kannappan, P.L. Quadratic functional equation and inner product spaces. Results Math. 1995, 27, 368-372. [CrossRef]

15. Bahyrycz, A.; Brzdęk, J.; Piszczek, M.; Sikorska, J. Hyperstability of the Fréchet equation and a characterization of inner product spaces. J. Funct. Spaces Appl. 2013, 2013. [CrossRef]

16. Brzdęk, J.; Leśniak, Z.; Malejki, R. On the generalized Fréchet functional equation with constant coefficients and its stability. Aequat. Math. 2018, 92, 355-373. [CrossRef] 
17. Chang, I.-S.; Kim, H.-M. Hyers-Ulam-Rassias stability of a quadratic functional equation. Kyungpook Math. J. 2002, 42, 71-86. [CrossRef]

18. Fechner, W. On the Hyers-Ulam stability of functional equations connected with additive and quadratic mappings. J. Math. Anal. Appl. 2006, 322, 774-786. [CrossRef]

19. Jung, S.M. On the Hyers-Ulam stability of the functional equations that have the quadratic property. J. Math. Anal. Appl. 1998, 222, 126-137. [CrossRef]

20. Kim, G.-H. On the stability of the quadratic mapping in normed spaces. Int. J. Math. Math. Sci. 2001, 25, 217-229. [CrossRef]

21. Malejki, R. Stability of a generalization of the Fréchet functional equation. Ann. Univ. Paedagog. Crac. Stud. Math. 2015, 14, 69-79. [CrossRef]

22. Sikorska, J. On a direct method for proving the Hyers-Ulam stability of functional equations. J. Math. Anal. Appl. 2010, 372, 99-109. [CrossRef]

23. Brzdęk, J.; Chudziak, J.; Páles, Z. A fixed point approach to stability of functional equations. Nonlinear Anal. 2011, 174, 6728-6732. [CrossRef]

24. Dung, N.V.; Hang, V.T.L. The generalized hyperstability of general linear equations in quasi-Banach spaces. J. Math. Anal. Appl. 2018, 462, 131-147. [CrossRef]

25. Cădariu, L.; Găvruta, L.; Găvruta, P. Fixed points and generalized Hyers-Ulam stability. Abst. Appl. Anal. 2012, 2012, 712743. [CrossRef]

26. Ciepliński, K. Applications of fixed point theorems to the Hyers-Ulam stability of functional equations-a survey. Ann. Funct. Anal. 2012, 3, 151-164. [CrossRef]

27. Gordji, M.E.; Khodaei, H. Solution and stability of generalized mixed type cubic, quadratic and additive functional equation in quasi-Banach spaces. Nonlinear Anal. 2009, 71, 5629-5643. [CrossRef]

28. Johnson, P.S.; Balaji, S. Hyers-Ulam stability of linear operators in Frechet spaces. Appl. Math. Inf. Sci. 2012, $6,525-528$.

29. Moraldlou, F.; Vaezi, H.; Eskandani, G.Z. Hyers-Ulam-Rassias stability of a quadratic and additive functional equation in quasi-Banach spaces. Mediterr. J. Math. 2009, 6, 233-248. [CrossRef]

30. Najati, A.; Eskandani, G.Z. Stability of a mixed additive and cubic functional equation in quasi-Banach spaces. J. Math. Anal. Appl. 2008, 342, 1318-1331. [CrossRef]

31. Najati, A.; Moghimi, M.B. Stability of a functional equation deriving from quadratic and additive functions in quasi-Banach spaces. J. Math. Anal. Appl. 2008, 337, 399-415. [CrossRef]

32. Xu, T.Z.; Rassias, J.M.; Xu, W.X. Generalized Hyers-Ulam stability of a general mixed additive-cubic functional equation in quasi-Banach spaces. Acta Math. Sin. 2012, 28, 529-560. [CrossRef]

33. Rolewicz, S. Metric Linear Spaces; PWN-Polish Scientific Publishers: Warsaw, Poland; D. Reidel Publishing Co.: Dordrecht, The Netherlands, 1984.

34. Piszczek, M.; Szczawaińska, J. Hyperstability of the Drygas functional equation. J. Funct. Space Appl. 2013, 2013, 912718. [CrossRef]

35. Piszczek, M.; Szczawaińska, J. Stability of Drygas functional equation on restricted domain. Results Math. 2014, 68, 11-24. [CrossRef]

36. Czerwik, S. On the stability of the quadratic mapping in normed spaces. Abh. Math. Sem. Univ. Hambg. 1992, 62, 59-64. [CrossRef]

(C) 2020 by the author. Licensee MDPI, Basel, Switzerland. This article is an open access article distributed under the terms and conditions of the Creative Commons Attribution (CC BY) license (http:/ / creativecommons.org/licenses/by/4.0/). 